


\title{
Sitzungsberichte
}

der

\section{Heidelberger Akademie der Wissenschaften}

\author{
Stiftung Heinrich Lanz \\ Mathematisch-naturwissenschaftliche Klasse
}

*) Jahrgang 1921 erschien im Verlage con Carl Winters Universitätsbuchhandlung in Heidelberg.

Im Verlag von Walter de Gruyter \& Co. vormals G.J. Göschen'sche Verlagshandlung - J. Guttentag, Verlagsbuchhandlung - Georg Reimer - Karl J.Trübner Veit \& Comp., Berlin erschienen:

Abteilung A. Mathematisch-physikalische Wissenschaften.

$$
\begin{aligned}
& \text { Jahrgang 1922: } 3 \text { Hefte. } \\
& \text { Jahrgang 1923: } 5 \text { Hefte. } \\
& \text { Jahrgang 1924. }
\end{aligned}
$$

1. Тн. Curtius und A. Bertho. Einwirkung von Stickstoffkohlenoxyd und von Stickwasserstoffsäure unter Druck auf aromatische Kohlenwasserstoffe. Reichsmark 0.50

2. Liebmann, Heinrich. Umkehrung des Variationsproblems der ebenen Affingeometrie. Reichsmark 0.60

3. Salomon, Wrlmelm. Die Intensitäten alluvialer und diluvialer geologischer Vorgänge und ihre Einwirkung auf die pliocäne Rumpfläche des Kraichgaves und Odenwaldes. Reichsmark 1.20

4. Heffeter, L. Zur absoluten Geometrie. Reichsmark 0.60

5. VAN WeRvEKE, L. Über die Entstehung der lothringischen Lehme und des mittelrheinischen Lößes. Reichsmark 1.50

6. KrULL, WolfaAxp. Die verschiedenen Arten der Hauptidealringe. Reichsmark 0.50

7. ROESER, ERnst. Übergang von der nichteuklidischen Streckentrigonometrie zur Winkelmessung. Reichsmark 0.30

8. Wellstein, Julius. Zur Differentialgeometrie der isotropen Kurven. Reichsmark 1.50

9. Ewald Rudolf. Die geodynamischen Ersoheinungen des krystallinen Odenwaldes als Beispiel einer geoisostatischen Ausgleichsschwingung. Reichsmark 1.50

10. Vortcker, Ilse. Uber eine ganz junge Verwerfung bei Rauenberg im Kraichgau. Reichsmark 0.30

11. Limbmans, Heinrich. Die Aufschliekung von Differentialinvarianten. Reichsmark 0.50

\section{Abteilung B. Biologische Wissenschaften.}

$$
\text { Jahrgang } 1923 .
$$

1. Kosser, A. und R. E. Gross. Über die Darstellung und quantitative Bestimmung des Arginins. Reichsmark 0.30

Von Jahrgang 1925 ab findet die Trennung in Abteilung $A$ und $B$ nicht mehr statt.

(Fortsetzung siehe 3. Umschlagseite)

*) Bestellungen auf solche Veröffentlichungen der math.-naturw. Klasse, welche früher im Verlag von Carl Winters Universitätsbuchhandlung in Heidelberg erschienen sind, nimmt auch der Verlag Walter de Gruyter \& Co., Berlin, entgegen. 